\title{
THE $p$-ADIC GAMMA MEASURES
}

\author{
JACK DIAMOND
}

\begin{abstract}
The $p$-adic log gamma function and its derivatives are used to define distributions and measures on the $p$-adic units. These measures are then used to interpolate the Leopoldt-Kubota $p$-adic $L$-functions on the positive integers.
\end{abstract}

1. Introduction. If $X$ is a compact-open subset of $Q_{p}$, a $p$-adic distribution on $X$ is an additive mapping from the compact open subsets of $X$ into $\Omega_{p}$. A bounded distribution is called a measure.

B. Mazur has used the Bernoulli polynomials $B_{k}$ to define an important family of distributions and measures on the $p$-adic integers. He then used the Bernoulli measures to express certain $p$-adic $L$-functions as integrals.

The Bernoulli distributions are defined for $k=0,1, \ldots$ by $\mu_{B, k}\left(a+p^{m} Z_{p}\right)$ $=p^{m(k-1)} B_{k}\left(a / p^{m}\right)$ where $a, m \in Z, m \geqslant 0,0<a<p^{m}$. The Bernoulli measures are defined for each $p$-adic unit $\alpha$ by $\mu_{k, \alpha}(A)=\mu_{B, k}(A)-$ $\alpha^{-k} \mu_{B, k}(\alpha A)$.

The purpose of this article is to use the $p$-adic log gamma function to define a set of distributions and measures which complement the Bernoulli distributions and measures.

The Bernoulli distributions are unique in the sense that the only polynomials $Q(X)$ that yield an additive function when used in the formula $\mu\left(a+p^{m} Z_{p}\right)=p^{m(k-1)} Q\left(a / p^{m}\right)$, are the constant multiples of the Bernoulli polynomials.

The gamma distributions satisfy this uniqueness property, if, instead of polynomials, we use functions of the form $Q(x)=\sum_{n=1}^{\infty} a_{n} / x^{n}$ and define $\mu$ on the $p$-adic units.

The Bernoulli measures are all related to $\mu_{1, \alpha}$ by the property

$$
\int_{X} 1 \cdot \mu_{k, \alpha}=\int_{X} k x^{k-1} \mu_{1, \alpha}
$$

This is analogous to the relation between $d x^{k}$ and $d x$ for $k$ a nonnegative integer. The gamma measures are the measures which relate to $\mu_{1, \alpha}$ as $d x^{k}$ relates to $d x$ with $k$ a negative integer.

Mazur found that the Bernoulli measures allow us to write $L_{p}(r, \chi)$ as an integral when $r$ is a negative integer. The integral has $r$ as a parameter, and is a continuous function of $r$ on the $p$-adic integers. Hence, by a continuity

Received by the editors April 26, 1978 and, in revised form, August 10, 1978.

AMS (MOS) subject classifications (1970). Primary 12B40; Secondary 12B30.

$K e y$ words and phrases. $p$-adic measures, log gamma function, $L$-functions. 
argument there is an integral formula for $L_{p}(r, \chi)$. The gamma measures allow us to calculate Mazur's integral when $r$ is a positive integer, so that we obtain the integral formula for $L_{p}(r, \chi)$ by interpolation across the positive integral values of $r$.

A description of Bernoulli measures and distributions can be found in Koblitz's book [4].

2. Notation. We will use $Q_{p}, Z, Z_{p}, Z_{p}^{x}$ and $\Omega_{p}$ for, respectively, the $p$-adic completion of the rational numbers, the ring of rational integers, the $p$-adic completion of $Z$ in $Q_{p}$, the units of $Z_{p}$ and the completion of the algebraic closure of $Q_{p}$. The $\log$ function is always the $p$-adic $\operatorname{logarithm}$ with $\log p=0$, as described by Iwasawa in [2].

If $a, b \in \Omega_{p}$, we will write $a \equiv b\left(\bmod p^{m}\right)$ to mean $|a-b|_{p}<p^{-m}$.

3. Gamma distributions. In order to define an additive function $\mu$ on the compact-open subsets of $Z_{p}^{x}$ it is sufficient (see [4]) to define $\mu$ on sets of the form $a+p^{m} Z_{p}$ with $a, m$ positive integers, $(a, p)=1,0<a<p^{m}$ and then check that

$$
\mu\left(a+p^{m} Z_{p}\right)=\sum_{b=0}^{p-1} \mu\left(a+b p^{m}+p^{m+1} Z_{p}\right)
$$

$\mu$ is then extended to all compact-open sets by

$$
\mu\left(\bigcup_{i=1}^{n} A_{i}\right)=\sum_{i=1}^{n} \mu\left(A_{i}\right), \quad A_{i} \cap A_{j}=\varnothing \text { if } i \neq j .
$$

Definition. For each nonnegative integer $k$ we define $\nu_{G, k}$ by $\nu_{G, k}(a+$ $\left.p^{m} Z_{p}\right)=p^{-k m} G_{p}^{(k)}\left(a / p^{m}\right)$, where $a, m \in Z, 0<a<p^{m},(a, p)=1$, and $G_{p}^{(k)}$ is the $k$ th derivative of the $p$-adic $\log$ gamma function $G_{p}[1]$.

The additivity of $\nu_{G, k}$ is a consequence of the Gauss multiplication formula for $G_{p}^{(k)}[1]$ :

$$
G_{p}^{(k)}(x)=p^{-k} \sum_{b=0}^{p-1} G_{p}^{(k)}\left(\frac{x+b}{p}\right) .
$$

A uniqueness theorem for the $\nu_{G, k}$ when $k>2$ follows easily from

Proposition 1. If

$$
f(x)=\sum_{n=k}^{\infty} \frac{a_{n}}{x^{n}}, \quad a_{n} \in \Omega_{p}, \quad a_{k} \neq 0, \quad k>1,
$$

is defined for $|x|_{p}>R \geqslant 1$, and

$$
f(p x)=\varepsilon \sum_{b=0}^{p-1} f\left(x+\frac{b}{p}\right)
$$

where $\varepsilon \in \Omega_{p}$ and $|x|_{p}>p R$, then $\varepsilon=p^{-k-1}$ and $f(x)$ is a constant multiple of $G_{p}^{(k+1)}(x)$. 
Proof. We have, by using the binomial expansion for $(x+b / p)^{-n}$,

$$
\sum_{b=0}^{p-1} f(x+b / p)=\sum_{m=k}^{\infty} \frac{1}{p^{m} x^{m}} \sum_{n=k}^{m} a_{n} p^{n} S_{m-n}\left(\begin{array}{c}
-n \\
m-n
\end{array}\right)
$$

where $S_{0}=p$ and $S_{r}=\sum_{b=0}^{p-1} b^{r}$.

Then, applying (1) and equating coefficients, we find that $a_{k}=\varepsilon a_{k} p^{k+1}$, so $\varepsilon=p^{-k-1}$ and, for $m>k, a_{m}$ is determined by

$$
a_{m}=\left(1-p^{-m-k}\right)^{-1} p^{-k-1} \sum_{n=k}^{m-1} a_{n} p^{n} S_{m-n}\left(\begin{array}{c}
-n \\
m-n
\end{array}\right) .
$$

Since $G_{p}^{(k+1)}(x)$ satisfies (1) it is clear from the recursion relation for the $a_{m}$ that $f(x)$ is a constant multiple of $G_{p}^{(k+1)}$.

It is not difficult to show that if $f(x)$ is given by a Laurent series for $|x|_{p}>R>1$, and $f(x)$ satisfies (1), then, up to a constant multiple, $f$ is either a Bernoulli polynomial $B_{k}(x)$ with $k \geqslant 0$ and $\varepsilon=p^{k-1}$ or $f(x)=G_{p}^{(k+1)}(x)$ with $k \geqslant 1$ and $\varepsilon=p^{-k-1}$.

The uniqueness result for $\nu_{G, k}$ is

THEOREM 1. If $\nu$ is a distribution on $Z_{p}^{x}$ with values in $\Omega_{p}$ and $\nu\left(a+p^{m} Z_{p}\right)$ $=\varepsilon^{m} f\left(a / p^{m}\right)$ where $a, m \in Z,(a, p)=1,0<a<p^{m}, \varepsilon \in \Omega_{p}$ and

$$
f(x)=\sum_{n=k}^{\infty} \frac{a_{n}}{x^{n}}, \quad a_{k} \neq 0, k \geqslant 1,
$$

then $\varepsilon=p^{-k-1}$ and $f(x)$ is a constant multiple of $G_{p}^{(k+1)}(x)$.

Proof. To satisfy the additivity condition, we need

$$
\begin{aligned}
\varepsilon^{m} f\left(\frac{a}{p^{m}}\right) & =\nu\left(a+p^{m} Z_{p}\right)=\sum_{b=0}^{p-1} \nu\left(a+b p^{m}+p^{m+1} Z_{p}\right) \\
& =\varepsilon^{m+1} \sum_{b=0}^{p-1} f\left(\frac{a}{p^{m+1}}+\frac{b}{p}\right) .
\end{aligned}
$$

Hence, $f(p x)=\varepsilon \sum_{b=0}^{p-1} f(x+b / p)$ for all $x$ of the form $p^{-m}$ with $m>1$. Since $\lim _{m \rightarrow \infty} p^{-m}=\infty$, the identity is valid for all $x$ with $|x|_{p}>p$.

4. Gamma measures. Mazur defined the Bernoulli measures $\mu_{k, \alpha}$ by the following formula. For each $\alpha \in Z_{p}^{x}$,

$$
\mu_{k, \alpha}(A)=\mu_{B, k}(A)-\alpha^{-k} \mu_{B, k}(\alpha A) .
$$

In a similar vein, we define the gamma measures $\nu_{k, \alpha}$ as follows:

$$
\begin{aligned}
& \nu_{0, \alpha}(A)=\nu_{G, 0}(A)-\alpha^{-1} \nu_{G, 0}(\alpha A)+((\log \alpha) / \alpha) \mu_{B, 1}(\alpha A), \\
& \nu_{1, \alpha}(A)=\nu_{G, 1}(A)-\nu_{G, 1}(\alpha A)+(\log \alpha) \mu_{B, 0}(A), \\
& \nu_{k, \alpha}(A)=\left((-1)^{k} /(k-2) !\right)\left(\nu_{G, k}(A)-\alpha^{k-1} \nu_{G, k}(\alpha A)\right) \text { for } k=2,3, \ldots
\end{aligned}
$$

It is clear from the definitions that each $\nu_{k, \alpha}$ is a distribution. The fact that the values of each $\nu_{k, \alpha}$ are bounded is implied by the following theorem. 
TheOREM 2. If $\alpha \in Z_{p}^{x}, a, m \in Z,(a, p)=1$ and $0<a<p^{m}$, then

(i) $\nu_{0, \alpha}\left(a+p^{m} Z_{p}\right) \equiv(\log a) \mu_{1, \alpha}\left(a+p^{m} Z_{p}\right)\left(\bmod p^{m}\right)$,

(ii) if $p>3, v_{1, \alpha}\left(a+p^{m} Z_{p}\right) \equiv(1 / a) \mu_{1, \alpha}\left(a+p^{m} Z_{p}\right)\left(\bmod p^{m}\right)$, if $p=2$, use $\bmod p^{m-2}$ and if $p=3$, use $\bmod p^{m-1}$,

(iii) if $p>3$ and $k \geqslant 2, v_{k, \alpha}\left(a+p^{m} Z_{p}\right) \equiv(1-k) a^{-k} \mu_{1, \alpha}\left(a+p^{m} Z_{p}\right)$ $\left(\bmod p^{m}\right)$.

If $p=2,3$, use $\bmod p^{m-1}$ instead of $\bmod p^{m}$.

Proof. We will show that, if $p>3$,

$$
\nu_{1, \alpha}\left(a+p^{m} Z_{p}\right) \equiv(1 / a) \mu_{1, \alpha}\left(a+p^{m} Z_{p}\right)\left(\bmod p^{m}\right) .
$$

The other results are proved in a similar manner.

For $x \in Z_{p}^{x}$ we define $\{x\}_{m}$ to be the unique positive integer $<p^{m}$ satisfying $\{x\}_{m} \equiv x\left(\bmod p^{m}\right)$, then $\left[x / p^{m}\right]$ is defined by

$$
\left[\frac{x}{p^{m}}\right]=\frac{x-\{x\}_{m}}{p^{m}}
$$

If $x \in Z$, then [ ] is just the usual "greatest integer" symbol.

From the definition we have

$$
\nu_{1, \alpha}\left(a+p^{m} Z_{p}\right)=p^{-m} G_{p}^{\prime}\left(\frac{a}{p^{m}}\right)-p^{-m} G_{p}^{\prime}\left(\frac{\{a \alpha\}_{m}}{p^{m}}\right)+\frac{\log \alpha}{p^{m}} .
$$

Now we use the Stirling series [1] valid for $|x|_{p}>1$,

$$
G_{p}(x)=\left(x-\frac{1}{2}\right) \log (x)-x+\sum_{r=1}^{\infty} \frac{B_{r+1}}{r(r+1) x^{r}},
$$

to obtain

$$
\begin{aligned}
\nu_{1, \alpha}(a & \left.+p^{m} Z_{p}\right) \equiv p^{-m}\left(\log \left(\frac{a \alpha}{\{a \alpha\}_{m}}\right)-\frac{p^{m}}{2 a}+\frac{p^{m}}{2\{a \alpha\}_{m}}\right)\left(\bmod p^{m}\right) \\
& \equiv p^{-m} \log \left(1+\frac{\left[a \alpha / p^{m}\right] p^{m}}{\{a \alpha\}_{m}}\right)+\frac{1}{2}\left(\frac{1}{a}\right)\left(\frac{1}{\alpha}-1\right)\left(\bmod p^{m}\right) \\
& \equiv \frac{1}{a}\left(\frac{1}{\alpha}\left[\frac{a \alpha}{p^{m}}\right]+\frac{1}{2}\left(\frac{1}{\alpha}-1\right)\right)\left(\bmod p^{m}\right)
\end{aligned}
$$

which, by definition of $\mu_{1, \alpha}$, (see [4])

$$
=(1 / a) \mu_{1, \alpha}\left(a+p^{m} Z_{p}\right) \text {. }
$$

- Theorem 2 not only shows each $\nu_{k, \alpha}$ is bounded, and therefore a measure, but also that $\nu_{1-k, \alpha}$ relates to $\mu_{1, \alpha}$ as $d x^{k}$ relates to $d x$ when $k$ is a negative integer. Furthermore, using $\log$ to mean real $\log$, we can say that $\nu_{0, \alpha}$ is analogous to $d(x(\log x)-x)$ and $\nu_{1, \alpha}$ is analogous to $d(\log x)$.

The analogies can be made precise with a corollary to Theorem 2 . 
Corollary. If $A$ is a compact-open subset of $Z_{p}^{x}$ and $f: A \rightarrow \Omega_{p}$ is continuous, then

(i) $\int_{A} f(x) \nu_{0, \alpha}=\int_{A} f(x)(\log x) \mu_{1, \alpha}$,

(ii) $\int_{A} f(x) \nu_{1, \alpha}=\int_{A}(f(x) / x) \mu_{1, \alpha}$,

(iii) if $k \geqslant 2, \int_{A} f(x) v_{k, \alpha}=\int_{A} f(x)(1-k) x^{-k} \mu_{1, \alpha}$.

Proof. By definition [4],

$$
\int_{A} f(x) v_{k, \alpha}=\lim _{m \rightarrow \infty} \sum_{\substack{a=0 \\ a+p^{m} Z_{p} \subset A}}^{p^{m}-1} f(a) \nu_{k, \alpha}\left(a+p^{m} Z_{p}\right) .
$$

The application of the congruences in Theorem 2 produces the corollary.

The following property of $\nu_{k, \alpha}$ is a direct consequence of the extension theorem for $G_{p}[1]: G_{p}(x)+G_{p}(1-x)=0$.

THEOREM 3. $v_{k, \alpha}=v_{k,-\alpha}$ for $k=0,1, \ldots$

5. Applications. We begin with the calculation of an integral.

Proposition 2. If $a, m, k \in Z,(a, p)=1,0<a<p^{m}$ and $k>2$, then

$$
\int_{a+p^{m} Z_{p}} t^{-k} \mu_{1, \alpha}(t)=\frac{(-1)^{k} p^{-m k}}{(k-1) !}\left(\alpha^{k-1} G_{p}^{(k)}\left(\frac{\{a \alpha\}_{m}}{p^{m}}\right)-G_{p}^{(k)}\left(\frac{a}{p^{m}}\right)\right) .
$$

Proof.

$$
\begin{aligned}
\int_{a+p^{m} Z_{p}} t^{-k} \mu_{1, \alpha}(t) & =\frac{1}{1-k} \int_{a+p^{m} Z_{p}} 1 \cdot v_{k, \alpha}=\frac{1}{1-k} \nu_{k, \alpha}\left(a+p^{m} Z_{p}\right) \\
& =\frac{(-1)^{k} p^{-m k}}{(k-1) !}\left(\alpha^{k-1} G_{p}^{(k)}\left(\frac{\{a \alpha\}_{m}}{p^{m}}\right)-G_{p}^{(k)}\left(\frac{a}{p^{m}}\right)\right)
\end{aligned}
$$

We can use Proposition 2 to obtain a formula for $G_{p}^{(k)}\left(a / p^{m}\right)$.

This formula has been found with a different technique by Koblitz [3].

THEOREM 4. If $a, m, k \in Z, p \nmid a, 0<a<p^{m}, k>2, \alpha \neq 1, \alpha \in Z_{p}^{x}$ and $\alpha \equiv 1\left(\bmod p^{m}\right)$, then

$$
G_{p}^{(k)}\left(\frac{a}{p^{m}}\right)=\frac{(-1)^{k} p^{m k}(k-1) !}{\alpha^{k-1}-1} \int_{a+p^{m} Z_{p}} t^{-k} \mu_{1, \alpha}(t) .
$$

Proof. Combine Proposition 2 with the observation that $\alpha \equiv 1\left(\bmod p^{m}\right)$ implies $\{a \alpha\}_{m}=a$.

We have a similar result for the values of $G_{p}\left(a / p^{m}\right)$. The proof is essentially the same as that of Theorem 4. 
THEOREM 5. If $a, m \in Z,(a, p)=1,0<a<p^{m}, \alpha \in Z_{p}^{x}, \alpha \neq 1$ and $\alpha \equiv 1\left(\bmod p^{m}\right)$, then

$$
G_{p}\left(\frac{a}{p^{m}}\right)=\frac{\log \alpha}{1-\alpha}\left(\frac{a \alpha}{p^{m}}-\frac{1}{2}\right)+\frac{\alpha}{\alpha-1} \int_{a+p^{m} Z_{p}}(\log t) \mu_{1, \alpha}(t) .
$$

Since $\nu_{k, \alpha}=\nu_{k,-\alpha}$ (Theorem 3), there are formulas for $G_{p}\left(a / p^{m}\right)$ and $G_{p}^{(k)}\left(a / p^{m}\right)$ when $\alpha$ is near -1 . Namely:

THEOREM 6. If $\alpha \equiv-1\left(\bmod p^{m}\right)$ and $a, m, k$ are as in Theorems 4 and 5 , then

$$
\begin{aligned}
G_{p}\left(\frac{a}{p^{m}}\right) & =\frac{-\log \alpha}{1+\alpha}\left(\frac{a \alpha}{p^{m}}+\frac{1}{2}\right)+\frac{\alpha}{\alpha+1} \int_{a+p^{m} Z_{p}}(\log t) \mu_{1, \alpha}(t), \\
G_{p}^{(k)}\left(\frac{a}{p^{m}}\right) & =\frac{(-1)^{k} p^{m k}(k-1) !}{(-\alpha)^{k-1}-1} \int_{a+p^{m} Z_{p}} t^{-k} \mu_{1, \alpha}(t) .
\end{aligned}
$$

$L$-FUnCTIONS. The integral in Proposition 2 can be used to do an interpolation of $L_{p}(r, \chi)$ on the positive integral values of $r$. We then obtain Mazur's formula for $L_{p}(r, \chi)$ when $\chi$ is a Dirichlet character $\bmod p^{m}$.

For $x \in Z_{p}^{x}$, let $\omega(x)$ be the unique $(p-1)$ th root of unity $\equiv x(\bmod p)$. Define $\langle x\rangle$ as $x \omega^{-1}(x)$. If $\chi$ is a Dirichlet character $\bmod p^{m}$, extend it to a mapping on $Z_{p}^{x}$ by letting $\chi(x)=\chi\left(\{x\}_{m}\right)$.

TheOREM 7 (MAZUR). If $\chi$ is a Dirichlet character $\bmod p^{m}, \alpha \in Z_{p}^{x}$, and $|r|_{p} \leqslant 1$, then

$$
\left(\langle\alpha\rangle^{r-1} \bar{\chi}(\alpha)-1\right) L_{p}(r, \chi)=\int_{Z_{p}^{x}} \frac{\chi(t)}{t\langle t\rangle^{r-1}} \mu_{1, \alpha}(t) .
$$

The left-hand factor on the left side is zero just when either $\alpha$ is a root of unity with $\chi(\alpha)=1$ or when $r=1$ and $\chi(\alpha)=1$.

Proof. We will use $\Sigma^{*}$ to indicate a sum in which the index of summation takes on only values not divisible by $p$.

If $r \in Z, r \geqslant 2$, then

$$
\begin{aligned}
\int_{Z_{p}^{*}} \frac{\chi(t)}{t\langle t\rangle^{r-1}} \mu_{1, \alpha}(t)=\sum_{a=1}^{p^{m}} \chi(a) \omega^{r-1}(a) \int_{a+p^{m} Z_{p}} t^{-r} \mu_{1, \alpha}(t) \\
=\sum_{a=1}^{p^{m}} * \frac{\chi(a) \omega^{r-1}(a)(-1)^{r} p^{-m r}}{(r-1) !}\left(\alpha^{r-1} G_{p}^{(r)}\left(\frac{\{a \alpha\}_{m}}{p^{m}}\right)-G_{p}^{(r)}\left(\frac{a}{p^{m}}\right)\right) \\
=\left(\langle\alpha\rangle^{r-1} \bar{\chi}(\alpha)-1\right) \frac{(-1)^{r} p^{-m r}}{(r-1) !} \sum_{a=1}^{p^{m}} \chi(a) \omega^{r-1}(a) G_{p}^{(r)}\left(\frac{a}{p^{m}}\right) \\
=\left(\langle\alpha\rangle^{r-1} \bar{\chi}(\alpha)-1\right) L_{p}(r, \chi) .
\end{aligned}
$$


This last step followed from comparing the definitions of $G_{p}^{(r)}(x)$ at $x=a p^{-m}[\mathbf{1}]$ and $L_{p}(r, \chi)[5]$ :

$$
\begin{aligned}
& G_{p}^{(r)}(x)=(-1)^{r}(r-2) ! \lim _{k \rightarrow \infty} \frac{1}{p^{k}} \sum_{n=0}^{p^{k}-1} \frac{1}{(x+n)^{r-1}} \\
& L_{p}(r, \chi)=\frac{1}{r-1} \lim _{k \rightarrow \infty} \frac{1}{p^{k}} \sum_{n=1}^{p^{k}} \frac{\chi(n)}{\langle n\rangle^{r-1}} \quad \text { if } \chi \text { is }\left(\bmod p^{m}\right) .
\end{aligned}
$$

Since each side of the equation in Theorem 7 is continuous on $Z_{p}$, and the integers $>2$ are dense in $Z_{p}$, the equality is valid for all $r \in Z_{p}$.

\section{REFERENCES}

1. J. Diamond, The p-adic log gamma function and p-adic Euler constants, Trans. Amer. Math. Soc. 233 (1977), 321-337.

2. K. Iwasawa, Lectures on p-adic L-functions, Ann. of Math. Studies, no. 74, Princeton Univ. Press, Princeton, N.J., 1972. MR 50 \# 12974.

3. N. Koblitz, Interpretation of the p-adic log gamma function and Euler constants using the Bernoulli measure, Trans. Amer. Math. Soc. 242 (1978), 261-269.

4. __ p-adic numbers, $p$-adic analysis, and zeta-functions, Graduate Texts in Mathematics, no. 58, Springer-Verlag, New York, 1977.

5. T. Kubota and H. W. Leopoldt, Eine p-adische THeorie der Zetawerte. I, J. Reine Angew. Math. 214/215 (1964), 328-339. MR 29 \#1199.

Department of Mathematics, Qubens Collegr (CUNY), Flushima, New York 11367 